\title{
Una investigación cualitativa: La creación del título de Enfermería en España (1915)
}

\section{Patricia Domínguez-Isabel ${ }^{1,2}$, Irene Soto-Fernandez ${ }^{1,3}$, Maria Mercedes de Dios-Aguado, 4 , María Angustias Torres-Alaminos $^{1,5}$, María Eva Moncunill Martínez ${ }^{1,6}$ y María Idoia Ugarte Gurrutxaga ${ }^{7}$}

\author{
${ }^{1}$ Estudio asociado HISAG-EP, Proyecto estructurante Historia y Epistemología, UICISA:E, \\ Coimbra, Portugal. \\ ${ }^{2}$ Hospital Virgen de la Salud, Toledo. Servicio de Urgencias, Sescam, España I \\ pdominguezi@sescam.jccm.es I https://orcid.org/0000-0001-6894-2270. \\ ${ }^{3}$ Escuela Pública, Ciudad de Nara. Avenida Francia 8, 45005, Toledo, España I \\ irene.soto.due@hotmail.com I https://orcid.org/0000-0002-1140-9544 \\ ${ }^{4}$ Centro de Salud de Yepes, Servicio de Salud de Castilla-La Mancha, Av. Santa Reliquia \\ 26, 45313, Toledo, España I mded@sescam.jccm.es I http://orcid.org/0000-0002-0991-7558 \\ ${ }^{5}$ Universidad de Castilla-La Mancha - Campus de Toledo, Departamento de Enfermería, \\ Fisioterapia y Terapia Ocupacional, Toledo. 45071, España I angustiast@gmail.com | \\ https://orcid.org/0000-0002-4523-5634 \\ ${ }^{6}$ Hospital Virgen de la Salud, Toledo, Espanha | mevamoncunill@hotmail.es \\ https://orcid.org/0000-0002-6885-530X \\ ${ }^{7}$ Universidad de Castilla-La Mancha, Espanha | Maria.Ugarte@uclm.es I \\ https://orcid.org/0000-0003-2413-3628
}

\begin{abstract}
Resumen: Introducción: El recorrido de historia de la enfermería desde una perspectiva de género permite comprender la vinculación de la enfermería con la figura femenina a través del tiempo y el progreso que experimentaron durante la primera mitad del siglo XX. En el marco teórico se establecen las bases del trabajo para llegar a comprender la importancia de movimiento feminista y su influencia en las leyes contra la discriminación por razones de género, estableciendo un enlace con la figura de la enfermera. Objetivos: Describir el proceso de la creación del título de Enfermería en España (1915) desde una perspectiva de género. Metodología: El presente estudio es una revisión histórico-narrativa realizada en tres fases y consultando las principales bases de datos como Dialnet, SciELO, MEDLINE/Pubmed, SciELO, CINAHL, CUIDEN, ScienceDirect. Resultados: El avance de la profesión enfermera se produce por el paso del ámbito doméstico al ámbito hospitalario desde el reconocimiento legal y científico. Gracias a la implicación de la Congregación de las Siervas de María en mayo de 1915 se reconoció la actividad profesional creándose el título oficial de Enfermería en España. Conclusiones: Este trabajo refleja las dificultades que supone traspasar las barreras de las mentalidades que componen a la sociedad y la falta de cambios a niveles políticos transversales a la sociedad y la cultura, dando así importancia a la investigación cualitativa para dejar reflejado estos hechos.
\end{abstract}

Palabras clave: Enfermería; Historia; Género; Mujer.

Qualitative research: The creation of the Nursing Degree in Spain (1915).

Summary: Introduction: A journey through the history of nursing from a gender perspective allows us to understand the link between nursing and the female figure over time and the progress experienced during the first half of the twentieth century. The theoretical framework establishes the basis of the work in order to understand the importance of the feminist movement and its influence on laws against gender discrimination, establishing a link with the figure of the nurse. Objectives: To describe the process of the creation of the Nursing degree in Spain (1915) from a gender perspective. Methodology: This study is a historical-narrative review carried out in three phases and consulting the main databases such as Dialnet, SciELO, MEDLINE/PubMed, SciELO, CINAHL, CUIDEN, ScienceDirect. Results: The advancement of the nursing profession is produced by the passage from the domestic sphere to the hospital sphere from the legal and scientific recognition. Thanks to the involvement of the Congregation of the Servants of Mary in May 1915, the professional activity was recognised and the official title of Nursing was created in Spain. Conclusions: This work reflects the difficulties involved in overcoming the barriers of mentalities that make up society and the lack of changes at transversal political levels in society and culture, thus giving importance to qualitative research in order to reflect these facts.

Keywords: Gender; History; Nursing; Women. 


\section{Introducción}

La historia ha sido construida por aquellos que tenían derecho a la educación y posibilidad de formación en diferentes campos y ciencias, por cuestiones de raza, sexo y posición social siendo así excluida una gran parte de la población. Por cuestión de sexo solo los hombres podían acceder a formación y dejaron plasmados escritos desde una visión donde predominaba la dominación masculina y dejaron a las mujeres en un plano inferior de sumisión. Con el concepto de género permite reescribir la realidad, visibilizando a las mujeres que formaron parte de la historia y que se mantuvieron silenciadas y olvidadas (Belmonte, 2008; García, 2016).

Como cita Robinson textualmente en el 1946 "la enfermera es el espejo en el que se reflejaba la situación de la mujer a través de los tiempos" (Donahue, 1985). Con lo cual este trabajo pretende analizar y reinterpretar la historia de la enfermería desde una perspectiva de género, y con ello, visibilizar al trabajo desempeñado por los/as profesionales dedicados/as al cuidado.

En la segunda mitad del siglo XIX, la enfermería en España estuvo marcada por una situación de progreso constante debido a que esta práctica sanitaria no estaba reconocida a nivel legal y social. Los cuidados ejercidos por este colectivo eran ligados directamente a la figura de la mujer y, por ende, a una figura sin recursos económicos, científicos y culturales (García \& Cravioto, 2013; Palanca de la Varga et al., 2017; Sánchez Miura \& Romero Castillo, 2017). Este trabajo era desarrollado dentro del domicilio del enfermo y se vinculó a un ideal de servicio doméstico donde los objetivos principales eran atender a las necesidades básicas del paciente como el aseo, el cuidado del bienestar, la alimentación, el sueño, la ayuda a la movilidad e incluso a la limpieza del domicilio (Espinosa, 2015; Sánchez et al., 2017).

Con la promulgación de la Ley de Bases Públicas, también conocida como Ley Moyano (1857), se legislaron las profesiones sanitarias (artículos 40 y 41) entre las que se encontraban las de practicante y matrona. El título de practicante dio la oportunidad de unificar una serie de oficios como sangradores, callistas, dentistas y asistentes a partos. El título de matrona recogió las profesiones de partera, comadrona y profesora de partos (Hernández-Martín, 2017; Martínez et al., 1998).

Aunque esta nueva ley diera cuerpo y forma a estas profesiones sanitarias dejó en el olvido a las mujeres que realizaban los cuidados básicos a pacientes que los precisaban en sus domicilios. A partir de este momento, las enfermeras quedaron casi cincuenta años sin un documento legal que reconociera su trabajo y con una formación sin regulación legal, es decir, no tenían acceso a unos conocimientos con un rigor científico ni un lugar y espacio donde llevarlo a cabo. Con tal situación, los cuidados de enfermería siguieron en manos de las órdenes religiosas y situadas en el ámbito hospitalario (Arroyo Rodríguez et al., 2011).

Durante la segunda mitad del siglo XIX y principios del XX la corriente ideológica que mantuvo a la mujer dentro de un prototipo específico fue la denominada domesticidad, influenciada por el tipo de pensamiento fruto de la sociedad patriarcal y religiosa. En el desglose de esta corriente encontramos prototipos e ideales donde la mujer era denominada con títulos como: El ángel del hogar, la perfecta casada o la mujer de su casa (Nash, 1994). Denominaciones que afianzaban y marginaban a la mujer a un ámbito doméstico, privado y cuyos motivos de realización vital se redujeron a la maternidad, la crianza y el cuidado del hogar. El concepto del Ángel del Hogar fue clave para continuar desarrollando un modelo decimonónico y es ahí donde se enmarcó la enfermera a principios del siglo XX (Barceló-Prats, 2018; Esteban, 2016).

El objetivo principal de este artículo ha sido describir la creación del título de enfermería en España en el 1915 desde una perspectiva de género. 


\section{Metodología}

La historiografía de la enfermería ha sido un reflejo de la historia de las mujeres, ya que la ligación del género femenino a esta profesión ha sido vinculante para la enfermería durante todo su recorrido profesionalizante. Junto con una perspectiva de género en este trabajo histórico permite una contribución crítica, y con ello el descubrimiento de más posibilidades de razonamiento y pensamiento al atender los diferentes factores vertebradores de los cuidados (Canalejo, 2013; Foth et al., 2018).

El presente trabajo es una revisión histórico-narrativa sobre la historia de la enfermería desde una perspectiva de género. Para ello se utilizó el modelo dialéctico estructural de cuidados (DSMC) ya que es una herramienta eficaz que ayuda a comprender las raíces sociales y culturales de las estructuras vinculadas a la división sexual y de género en el trabajo, comprendiendo de esta manera las causas por las que se producen los cambios en la sociedad (González \& Ruiz, 2016; Kuerten Rocha \& Lenise do Prado, 2008).

Las estructuras que se aplican son 1) unidad funcional (UF), incluye normas, valores, creencias, conocimientos y sentimientos que dan origen a los sistemas sociales y que determinan el género y la división sexual del trabajo, tan decisivos en el surgimiento de estas profesiones donde lo fisiológico se fusiona con el trabajo; 2) Marco funcional (MF), relacionado con el lugar donde se realizan las actividades, 3) elemento funcional (EF), incluye los actores sociales, responsables de la gestión de los cuidados. Estos constituyen una herramienta adecuada para la ordenación y análisis de datos para obtener una visión global de los fenómenos históricos para la perspectiva de la historia cultural (Siles, 2016; Siles-González et al., 2020).

\section{Modelo Estructural Dialéctico de los Cuidados (MEDC)}

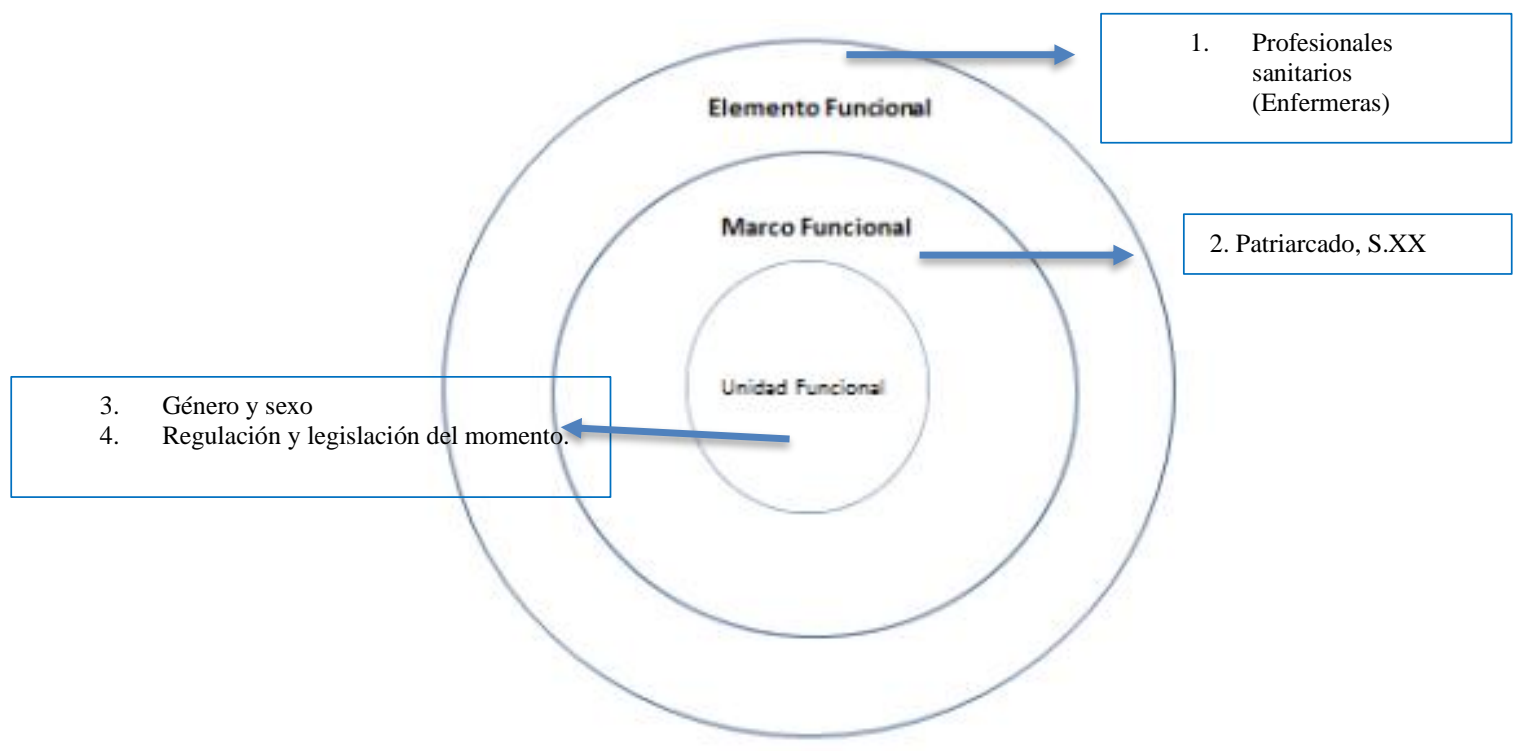

Fig. 1. Siles, J. (2010). Historia cultural de enfermería reflexión epistemológica y metodológica. Avances en enfermería. 18: 120-128.

UF:

Las distinciones entre mujeres y hombres de acuerdo con sus diferencias biológicas, es decir, por el sexo, cuentan con un recorrido histórico amplio. Cada sexo está determinado por unas características físicas, biológicas y fisiológicas adquiridas de manera natural. Pero estas diferencias se han visto condicionadas e interpretadas erróneamente en base a justificaciones ligadas a factores sociales, culturales, políticos, psicológicos, jurídicos y económicos, dando lugar a una serie de roles estereotipados que, durante mucho tiempo, han asumido patrones inflexibles de comportamiento. 
Se trata, por tanto, de un conjunto de construcciones socioculturales que han dado forma al género femenino o masculino, y actualmente, también a una diversidad dentro de estas categorías. Como todo esto es inherente y transversal a la cultura, los estereotipos de género se adquieren por un proceso de socialización primaria y secundaria mediante la educación, el lenguaje, los estereotipos, la religión y los símbolos (Arroyo Rodríguez et al., 2011; Pinzón Estrada et al., 2017).

Marta Lamas, con su teoría de la construcción de la identidad desarrollada en 1986, explica que en un principio hay una asignación de sexo según el conocimiento genital. Más tarde, en torno a los tres años se produce un reconocimiento de la identidad "yo soy hombre" o "yo soy mujer". El aprendizaje de estas diferencias permite su generalización a otras situaciones, que van desde, la forma de vestir o la división de los juguetes, hasta las relaciones entre la familia y el contexto social. Más tarde se adquiere un papel o rol en la sociedad y en consecuencia la existencia de una división de trabajos por sexos (Lamas, 2018).

MF:

Dentro de un entorno los comportamientos son aprendidos y en este caso existe la dominación masculina, es decir, donde las sociedades son patriarcales, y el mundo sanitario ha sido y es un reflejo de esto (Canalejo, 2013). Inicialmente, el concepto de patriarcado fue introducido por Kate Millet, señalándolo como una herramienta analítica donde explicaba las relaciones de género de una manera asimétrica en las distintas estructuras sociales y producciones históricas, que funcionan como un opresor de las mujeres, ya que las relega al desarrollo de actividades domésticas no reconocidas y en el ámbito privado (de García Carraso, 1974). Con la existencia de una latente creencia de superioridad del hombre, que cuenta con un mayor poder a diferentes niveles sociales y culturales, ejerce un rol dominante sobre la mujer haciéndola creer que le debe subordinación y obediencia por razón de su naturaleza.

EF:

Todo esto lo encontramos plasmado en la situación de los/as profesionales de la salud en el 1915, cuando las mujeres comenzaron a querer formar parte del mundo laboral y a perseguir la formación como enfermeras en espacios públicos (universidades y/o colegios). A pesar de que la Universidad como institución abrió las puertas a las mujeres, médicos y practicantes se negaron ya que alegaban encontrar inapropiado que ellas tuvieran importancia o visibilidad en el ámbito sanitario (García, 2016).

Gayle Rubin señala que esta discriminación sexual del trabajo tiene su repercusión a la hora de ejercer en un ámbito público o privado, siendo este último sinónimo de doméstico y ahí encontramos enmarcada la figura de la enfermera en la última mitad del siglo XIX y principios del XX (Hendel, 2017; Lamas, 2018).

\subsection{Estrategia de Búsqueda.}

Se ha realizado una búsqueda de documentos en base al tema de estudios siguiendo una serie de fases entre los meses de septiembre de 2020 y febrero de 2021. Durante la primera fase se realizó una búsqueda en las bases de datos disponibles en la red: Medical Literature Analysis and Retrieval System Online (MEDLINE/PubMed), Dialnet, SciELO, Cumulated Index of Nursing \& Allied Health Literatura (CINAHL), CUIDEN, ScienceDirect y Google Académico; en la segunda fase se ejecutó una exploración a través de los servicios y recursos disponibles en la Biblioteca online, Plinio y con acceso directo a través la biblioteca de la Universidad de Castilla-La Mancha; en la tercera fase se consultaron páginas web, como la de El Fondo de las Naciones Unidas para la Infancia (UNICEF), en la página de la Organización Mundial de la Salud (OMS) y en el Ministerio de Sanidad.

Los descriptores que se emplearon para realizar esta búsqueda en las diversas bases de datos fueron palabras claves en los idiomas de español e inglés, estas fueron: "perspectiva de género" "perspective of gender", "enfermería", "nursing", "historia", "history". 
Las fórmulas empleadas para el tema de estudio fueron: (history of nursing) AND perspectiva of gender y ((perspective of gender) AND nursing) AND history.

Como criterios de inclusión se han seleccionado 1) artículos, libros y capítulos de libros a texto completo; 2) idiomas de tema seleccionado como español e inglés; 3) documentos con alta relevancia científica, donde se incluyen libros, manuscritos y artículos de revista; 4) bibliografía de no mayor antigüedad a los 10 años de publicación, salvo aquella considerada relevante e importante para el tema de estudio.

Tras la búsqueda, revisión y selección de fuentes utilizadas, se ha llegado a elegir un total de 19 artículos de revista y 7 libros y/o capítulos de libro.

\section{Diagrama de flujo}

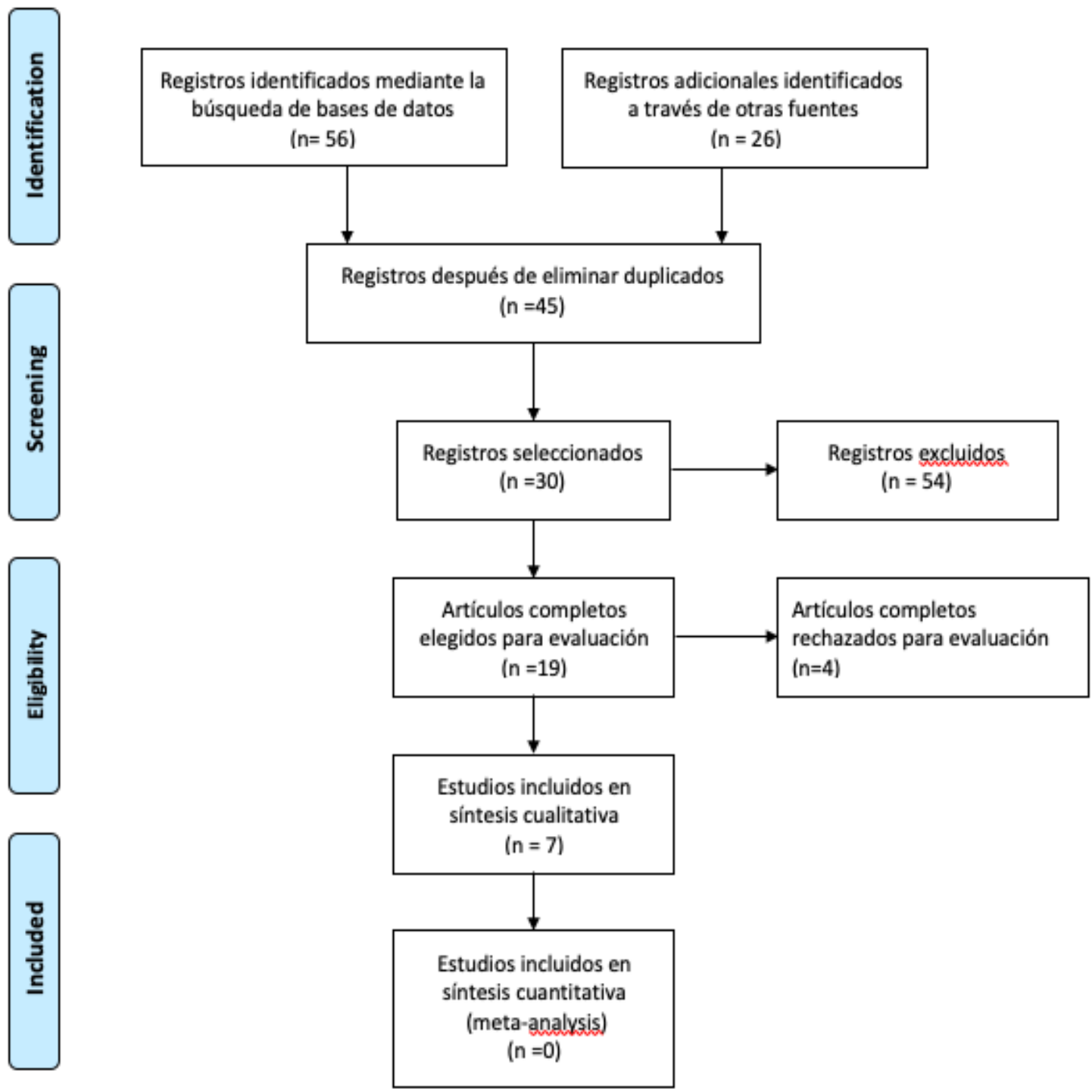

Fig. 2. Moher D, Liberati A, Tetzlaff J, Altman DG, The PRISMA Group (2009). Preferred Reporting /tems for Systematic Reviews and Meta-Analyses: The PRISMA Statement. PLoS Med 6(7): e1000097.

doi:10.1371/journal.pmed1000097 
Vol. 8 | Investigación Cualitativa en Salud: Avances y Desafíos

Tabla 1. Bibliografía consultada, bases de datos y palabras claves,

\begin{tabular}{cccc}
\hline Bases de datos & Palabras Clave & Documentos consultados & $\begin{array}{c}\text { Documentos } \\
\text { seleccionados }\end{array}$ \\
\hline Dialnet & & 10 & 3 \\
SciELO & "Enfermería"; "Historia"; & 15 & 4 \\
CUIDEN & "Mujer"; "género"; "Siglo & 8 & 3 \\
CINAHL & XX"; "perspectiva de & 16 & 1 \\
PubMed & género". & 5 & 2 \\
Base UCLM & & 10 & 3 \\
Página Webs & & 4 & 1 \\
Periódicos & & 5 & 19 \\
TOTAL & & 73 &
\end{tabular}

Tabla 2. Bibliografía consultada, libros y capítulos de libro.

\begin{tabular}{cccc}
\hline $\begin{array}{c}\text { Libros y/o capítulos } \\
\text { de libro }\end{array}$ & Palabras Clave & $\begin{array}{c}\text { Documentos } \\
\text { Consultados }\end{array}$ & $\begin{array}{c}\text { Documentos } \\
\text { seleccionados }\end{array}$ \\
Biblioteca UCLM & $\begin{array}{c}\text { "Enfermería"; } \\
\text { "Historia"; "Mujer"; } \\
\text { "género"; "Siglo XX". }\end{array}$ & 23 & 7 \\
\hline
\end{tabular}

\section{Resultados}

A finales del siglo XIX la lucha del movimiento feminista en España perseguía conseguir la igualdad entre hombres y mujeres influenciado por Emilia Pardo Bazán junto con Concepción Arenal. Ambas establecieron objetivos que buscaban romper con el estereotipo impuesto a las mujeres, el cual perseguía conseguir la figura denominada ángel del hogar, para dejarlo atrás y obtener así independencia en la toma de decisiones y acceso a la educación (Lagunas, 2017).

El contexto social está marcado por algunos aspectos como son la organización sociopolítica del momento y los cambios sociales que afectan a toda la población. En esta época el sistema constitucional de España (1900-1920) era una monarquía doctrinaria y no democrática. Esto quiere decir que el rey Alfonso XIII tenía el poder absoluto del estado y por consecuente de los tres poderes políticos: legislativo, jurídico y legal. La política controlaba los diferentes aspectos de la vida, entre ellos la vida de las mujeres, mediante leyes que regulaban la educación y el trabajo. Por ello, las mujeres eran situadas en un plano de inferioridad y en desigualdad de oportunidades desde un marco legal, tanto para acceder al mundo laboral como a la educación (López Vallecillo, 2016).

En un marco internacional, los Estados Europeos desempeñaron un papel relevante puesto que crearon leyes basadas en el discurso de género que garantizaban normas legales, educativas y política de desigualdad y diferenciación entre los roles a desempeñar por mujeres y hombres. El Código Napoleónico es un claro ejemplo de esto, reflejado en nuestro país en el Código Civil y en el Penal. Dejaban a las mujeres en un segundo plano subordinabas al hombre una vez que se contraía matrimonio, ya que por ley debían obediencia a su marido y siempre necesitarían de su consentimiento para participar en todos los actos públicos como pudieran ser pleitos, compras y ventas. 
Es entonces donde el feminismo, categorizado como una corriente del pensamiento con gran influencia en la edad contemporánea, comienza a transformar la visión de las mujeres (Espinosa, 2015).

La construcción social de lo que era ser mujer en esta época estaba totalmente alineada con el arquetipo llamado ángel del hogar, definido y caracterizado por una mujer que desempeñaba de una manera ejemplar los roles de madre y esposa, desde valores como el del sacrificio por la familia. En este estereotipo, la mujer tenía su realización personal en la maternidad y cuidado del hogar, es decir, era imperante la idea de la domesticidad. Características como la inteligencia y el trabajo eran asignadas directamente a los hombres, mientras que a la mujer se la definía por tener sentimientos de afectividad, dulzura y abnegación. Esto reforzaba aún más la idea de que las mujeres por su naturaleza estaban destinada a permanecer en el hogar y a ser las cuidadoras principales de la familia (Pinzón Estrada et al., 2017).

Durante el reinado de Alfonso XIII, la mujer comienza a luchar por romper con esta idea para conseguir su emancipación y separación impuesta por la sociedad patriarcal, donde dejaba siempre a la figura de la mujer marginada en la esfera privada. En esta época comienzan a aparecer una serie de grupos femeninos que lucharon por la plena integración de la mujer en la vida política y social. Se crearon varias asociaciones de mujeres en la primera mitad del siglo XX, con el objetivo de dar fuerza, como La Asociación Nacional de Mujeres Españolas (ANME) o El Lyceum Club Femenino. Ambas querían buscar la ruptura del rol tradicional femenino, conseguir el voto, reformar el código civil y la igualdad femenina (López Vallecillo, 2016).

En estos primeros años del siglo, con el auge de la industrialización, el trabajo de las mujeres podía estar o no remunerado pero la gran mayoría de ellas desempeñaban trabajos domésticos, como podía ser el de enfermera y en el ámbito rural la agricultura. Aunque hubo mujeres trabajadoras en fábricas, éstas tenían un salario inferior al de los hombres a pesar de realizar las mismas funciones y jornadas, existía por tanto una discriminación sexual en el trabajo. Pero de esta manera se consiguió que las mujeres pudieran liberar un trabajo fuera del hogar. No obstante, procesos naturales como el embarazo, la lactancia y los cuidados de los hijos e hijas ocupaban el mayor tiempo de estas mujeres, siendo todavía objetivos principales en sus vidas.

A su vez, en función de la clase social que ocuparan las mujeres, su vida era enfocada de diferente manera existiendo una variabilidad de metas a alcanzar. Por tanto, las relaciones establecidas son jerarquizaciones sociales, donde la cultura androcéntrica imponía y definía que roles estereotipados tenía que desarrollar cada sexo (Cobo Bedia et al., 2009).

En particular el papel de la enfermería nació y creció en este contexto como una nueva profesión que permitió a la mujer conseguir salir del hogar, con una formación reglada y un trabajo remunerado. En este momento estaba en una gran desventaja a nivel de reconocimiento social y valor científico situándola en una posición inferior comparado con carreras universitarias como medicina 0 títulos como el de practicante, ambas desarrolladas por hombres. En consecuencia, la profesión de enfermería estuvo feminizada durante muchos años y por ende desvalorada con una notable discriminación laboral y con presencia en su desarrollo de un sexismo ambivalente. Para poder entender cómo nació el título de enfermería es necesario realizar un recorrido histórico previo (Espinosa, 2015).

\section{Conclusiones}

La Historia es una ciencia compleja que recopila los sucesos y acontecimientos del ser humano a su paso por la Tierra, permitiendo además su trasmisión generación tras generación. Esta, aunque pretende ser fidedigna a los hechos y objetiva, en muchos casos no es así, ya que hay aspectos de las vidas de las personas y de su conjunto como sociedad que no han sido el foco de atención de esta ciencia y, por tanto, han quedado en el olvido. 
En relación con el objetivo principal de este trabajo, el desarrollo de la disciplina enfermera fue una carrera larga, repleta de dificultades y barreras, pero que con resistencia y esfuerzo las enfermeras pudieron llegar a la meta obteniendo el reconocimiento social que se merecían. Este trabajo se ha abordado desde una perspectiva de género, ya que a mi manera de ver la historia y quien le ha escrito, ha dejado a un lado los sucesos relacionados con las mujeres, sin visibilidad hasta nuestros días. Por ello, con este estudio de revisión bibliográfica pongo de relieve los aspectos tanto como mujer y enfermera que han hecho que hoy día pueda llegar a convertirme en lo que he querido ser.

Tal situación no deja de reflejar la dificultad en el cambio de mentalidades, que no sólo puede venir de la mano de una parte de la estructura social, de la profesión, sino que requiere de factores políticos transversales a la sociedad y la cultura. Se puede observar cómo en este momento histórico, el contexto socioeconómico y político vertebran la situación de la mujer a través de los principales ejes de desigualdad, en este caso, el género, la edad, el territorio y, en cierta medida, la clase social.

La investigación cualitativa ayuda a mantener la coherencia entre los objetivos, teoría y método, así como un enlace y compresión de los resultados obtenidos, dando una lógica científica a los hechos surgidos y una continuidad entre ellos. Además, constituye una herramienta eficaz para organizar los datos recogidos obteniendo enfoques y análisis de los datos para conseguir visiones globales de los diferentes momentos históricos.

\section{Agradecimientos}

A nuestro grupo de investigación HISAG-EP. Este estudio no ha sido financiado por ninguna entidad y no existen conflicto de intereses entre los autores.

\section{Referencias}

Arroyo Rodríguez, A., Lancharro Tavero, I., Romero Serrano, R., \& Morillo Martín, M. S. (2011). La Enfermería como rol de género. Index de Enfermería, 20(4), 248-251. https://doi.org/10.4321/S1132-12962011000300008

Barceló-Prats, J. (2018). Relaciones de la Enfermería con la Antropología Médica: Bases para una historia antropológica de la Enfermería hospitalaria. En Poder e influencia de las enfermeras en la Historia (Col-legi Oficial d' Infermeria de les Illes Balears, pp. 83-99).

Belmonte, C. R. (2008). Concepto de género: Reflexiones. Ensayos: Revista de la Facultad de Educación de Albacete, 23, 307-314.

Canalejo, C. G. (2013). Análisis histórico de la situación de las enfermeras y matronas en españa.: Una perspectiva global. Raudem: Revista de estudios de las mujeres, 1, 50-65.

Cobo Bedia, R., Cruz, C. de la, Volio, R., Zambrano, A., Aparicio García, M., Leyra Fatou, B., \& Ortega Serrano, R. (2009). Cuadernos de género: Políticas y acciones de género. Materiales de formación. Instituto Complutense de Estudios Internacionales (ICEI).

De García Carraso, C. A. (1974). La emancipación de la mujer en España (Vol. 18). Júcar.

Donahue, M. P. (1985). Historia de la Enfermería. Barcelona [etc.] : Doyma. https://catalogobiblioteca.uclm.es/cgi-bin/abnetopac/O7135/IDa67334e7/NT8

Espinosa, M. C. (2015). Centenario de la creación del título de enfermera en España: Una mirada a las mujeres que prestan cuidados. VII Congreso virtual sobre Historia de las Mujeres, 2015, págs. 4558, 45-58. https://dialnet.unirioja.es/servlet/articulo?codigo $=5346826$

Esteban, N. A. (2016). El ángel del hogar y sus demonios. Ciencia, religión y género en la España del siglo XIX. Historia Contemporánea, 0(21). https://www.ehu.eus/ojs/index.php/HC/article/view/15898

Foth, T., Lange, J., \& Smith, K. (2018). Nursing history as philosophy-towards a critical history of nursing. Nursing Philosophy: An International Journal for Healthcare Professionals, 19(3), e12210. https://doi.org/10.1111/nup.12210 
García, A. G. (2016). La actividad de las matronas de la Beneficencia Municipal en Cuenca (España) a comienzos del siglo XX desde la perspectiva de género. HiSTOReLo. Revista de Historia Regional y Local, 8(16), 228-269.

García, I. G., \& Cravioto, E. G. (2013). Surgimiento y desarrollo de la Historia de la Enfermería en España. Enfermería Global, 12(2). https://doi.org/10.6018/eglobal.12.2.160381

González, J., \& Ruiz, C. (2016). El Modelo Estructural Dialéctico de los Cuidados. Una guía facilitadora de la organización, análisis y explicación de los datos en investigación cualitativa. CIAIQ2016, 2. https://proceedings.ciaiq.org/index.php/ciaiq2016/article/view/754

Hendel, L. (2017). Comunicación, infancia y adolescencia: Guía para periodistas (Primera Edición). Fondo de las Naciones Unidas para la Infancia (UNICEF). www.unicef.org.ar

Hernández Martín, F. J. (2017). Relaciones interprofesionales practicantes/religiosos: Una aproximación al tema. En Los Colegios Profesionales de Enfermería, su función social e institucional. (pp. 187-195). Colegio Profesional de Enfermería de Salamanca.

Kuerten Rocha, P., \& Lenise do Prado, M. (2008). Modelo de Cuidado: ¿Qué es y como elaborarlo? Index de Enfermería, 17(2), 128-132.

Lagunas AC. La Falange es un modo de ser (mujer): discursos e identidades de género en las publicaciones de la Sección Femenina (1938-1945). Hist Política Ideas Procesos Mov Soc. 2017;(37):91-120.

Lamas, M. (2018). El género: La construcción cultural de la diferencia sexual. El género, 1-366.

López Vallecillo, M. (2016). Presencia social e imagen pública de las enfermeras en el siglo XX (19151940) [Instituto de Historia Simancas]. http://uvadoc.uva.es/handle/10324/16513

Martínez, G., Jesús, M., \& Martínez, A. C. G. (1998). Fechas claves para la historia de las Matronas en España. Génesis, 38, 27-30.

Nash, M. (1994). Experiencia y aprendizaje: La formación histórica de los feminismos en España. Historia social, 20, 151-172.

Palanca de la Varga, J. M., Pérez García, P., \& Castro González, M. P. (2017). Evolución de enfermería en España en el siglo XIX. Institucionalización de la enfermería como profesión. En Los Colegios Profesionales de Enfermería, su función social e institucional. (pp. 333-341). Colegio Profesional de Enfermería de Salamanca.

Pinzón Estrada, C., Aponte Valverde, M. V., \& Useche Morillo, M. L. (2017). ¿Sexismo en enfermería? Una mirada desde la perspectiva de género a roles feminizados como el cuidado. Sexism in Nursing? A look from the gender perspective to feminized roles such as care., 23, 123-146. https://doi.org/10.25100/prts.v0i23.4590

Sánchez Miura, J. A., \& Romero Castillo, R. (2017). La enfermería durante el reinado de Isabel II. Marco Legislativo de la profesionalización enfermera en España. En Los Colegios Profesionales de Enfermería, su función social e institucional. (pp. 313-318). Colegio Profesional de Enfermería de Salamanca.

Sánchez, Y. M. M., Daza, M. F., Acuña, A. I., \& Restrepo, S. S. (2017). Cronología de la profesionalización de la Enfermería. Revista Logos, Ciencia \& Tecnología, 9(2), 63-83.

Siles, J. (2016). Teoria e Metódo nos Estudos Históricos em Enfermagem - o Modelo Estrutural Dialético dos Cuidados (MEDC)En: Oguisso, T., Freitas, G.F. y Siles, J. (Eds.), Enfermagem: História, Cultura dos Cuidados e Métodos (pp.291-317). Río de Janeiro: Águia Dourada. (pp. 291-317).

Siles-González, J., Romera-Álvarez, L., Dios-Aguado, M., Ugarte-Gurrutxaga, M. I., \& Gómez-Cantarino, S. (2020). Woman, Mother, Wet Nurse: Engine of Child Health Promotion in the Spanish Monarchy (1850-1910). International Journal of Environmental Research and Public Health, 17(23), 9005. https://doi.org/10.3390/ijerph17239005 